\title{
Piezoelectric transceiver matching for multiple frequencies
}

\author{
A. Ens and L. M. Reindl \\ Laboratory for Electrical Instrumentation, University of Freiburg, Freiburg, Germany \\ Correspondence to: A. Ens (alexander.ens@imtek.uni-freiburg.de)
}

Received: 11 August 2014 - Revised: 20 November 2014 - Accepted: 5 December 2014 - Published: 12 January 2015

\begin{abstract}
Robust data transmission over long ranges with standard ultrasound devices is a challenge. Ultrasound indoor positioning systems in particular require long ranges and a robust data communication link. Fundamentally, a piezoelectric transducer has a narrow bandwidth for high sound pressure level and efficiency and is not suitable for broad-band applications. Moreover, ultrasound attenuation in the air increases quadratically within frequency, and thus ultrasound localization systems are restricted to low frequencies and low bandwidths.

This work presents a novel method to match a piezoelectric transceiver for multiple frequencies by using the parallel and the series resonance of the transceiver. The aim is to adjust the amplitudes at different frequencies from different senders to the same level, which is important for orthogonal frequency division multiplex communication systems. Hence, an analog-to-digital converter (ADC) with low dynamic range (low voltage resolution) can be used to measure multiple frequencies with the same resolution. As a result, the optimization decreases the required dynamic range by $6 \mathrm{~dB}$. Consequently, the $\mathrm{ADC}$ requires 1 bit fewer to ensure the same resolution for all carrier frequencies.
\end{abstract}

\section{Introduction}

Ultrasound seems to be a preferable technology for indoor localization. It is low cost, the speed of sound is about six decades slower than the speed of light and it is limited to closed rooms. Lerch et al. (2008) and Tränkler and Reindl (2014) presented systems for distance measurements and object recognition. Hence, high localization accuracy can be achieved with low complexity devices and a low sampling frequency analog-to-digital converter (ADC) due to the low velocity of sound propagation. Furthermore, the sound distribution is limited to small areas (e.g., rooms). However, the expected maximal distance for ultrasound is about $20 \mathrm{~m}$ and the bandwidth is small compared to radio frequency-based systems.

Piezoelectric transducers have a high quality factor $Q$ and high sound pressure levels (SPL) up to $120 \mathrm{~dB}$ (in $0.2 \mathrm{~m}$ distance at only $U_{\mathrm{pp}}=20 \mathrm{~V}$ ), whereas the usable bandwidth is small and enables only narrow bandwidth application. Hence, Ens et al. (2013) developed a modulation scheme that is based on frequency shift keying (FSK) and chirp spread spectrum (CSS) techniques to achieve robust data transmission with narrow bandwidth devices. However, Jakes (1993) showed that multi-carrier data communication provides higher spectral efficiency than CSS modulation. Therefore, we are interested in adapting the system for multi-carrier data communication in an orthogonal frequency division multiplex (OFDM). Chang (1966) and Weinstein and Ebert (1971) showed the possibilities and advantages of OFDM. Transmission systems require transducers with higher bandwidth or the use of multiple transducers with different resonance frequencies. Hosman et al. (2011) showed the use of a standard $40 \mathrm{kHz}$ piezoelectric ultrasound transducer for data communication in metal within a frequency band of 260 to $330 \mathrm{kHz}$. Providing such high frequencies to ultrasound, the signal $(200 \mathrm{kHz})$ will be attenuated by about $60 \mathrm{~dB}$ (about $33 \mathrm{~dB}$ for $40 \mathrm{kHz}$ ) at a $5 \mathrm{~m}$ distance (ISO, 1993). However, we use lower frequencies $(40 \mathrm{kHz})$ for our data transmission to reduce the attenuation by air.

Oralkan et al. (2002) and Caliano et al. (2010) investigated the bandwidth of capacitive micro-machined ultrasonic transducers (CMUT) and showed the broad-band capabilities. They have a wide-band characteristic and can be 
fabricated with standard silicon technology. Furthermore, the CMUTs are designed to operate below the resonance frequency of the device. This enables higher bandwidths. CMUTs include a low noise amplifier (LNA) to gain the signal. Hence, the device requires no matching and the signal can be amplified with an operational amplifier. However, the noise floor of the CMUT devices is higher than for piezoelectric devices. Furthermore, Anderson et al. (2005) showed that the CMUTs require high voltages to transmit signals and they have a low efficiency.

Bloomfield et al. (2000) presented ultrasound transducers based on polyvinylidene fluoride (PVDF) with high bandwidth and a relativly good acoustic impedance match to water. However, they only have an output of about $0.025 \mathrm{~Pa} \mathrm{~V}^{-1}$ and require a supply voltage of about $200 \mathrm{~V}_{\mathrm{pp}}$ to achieve a SPL of $107 \mathrm{~dB}$; one example is the US40KT-01 transducer by MSI.

Ealo et al. (2008) showed the use of a microporous polypropylene foam with permanent charge and a broadband characteristic as an electromechanical film (EMFi). The transducer is customizable and consists of a film glued to a rigid surface and the connections for an external supply voltage. However, there are no commercially available EMFitransducers and the high supply voltage makes it unsuitable for low-power application.

Manufacturers provide ultrasonic transducer in pairs for sending and receiving the signal. They design the device so that the sender has a series resonance near the same frequency as the parallel resonance of the receiver. The presented work shows how to use both transducers for sending on two frequencies and only one transducer for receiving the signal. Moreover, it shows an optimization method with a measurement resistor to use for a series and a parallel resonance circuit for multi-frequency applications. This includes the calculation of the measurement resistor parallel to the piezoelectric transducer. The transmission system receives two signals with two different frequencies, which are also generated with piezoelectric transducers. The aim of the optimization is to match both signals to the same amplitude. Hence, a low-cost ADC with lower dynamic range can be used to convert the signals to digital values.

\section{Model}

The system is designed to transmit data over two separate channels with different carrier frequencies in OFDM. Figure 1 shows the schematic diagram of the transmission system. The data bits $\left(b_{0}\right.$ and $\left.b_{1}\right)$ are mapped by $\pi / 4$-BSK (binary shift keying; Höher, 2013), and the pulse width modulation (PWM) generates the analog signal. Additionally, the power amplifiers (PA) gain the signal before the piezoelectric transducers transform the electric signal to ultrasound signal. The composite signal traveling within air medium is transformed to electric signals at the receiver by the piezoelec-

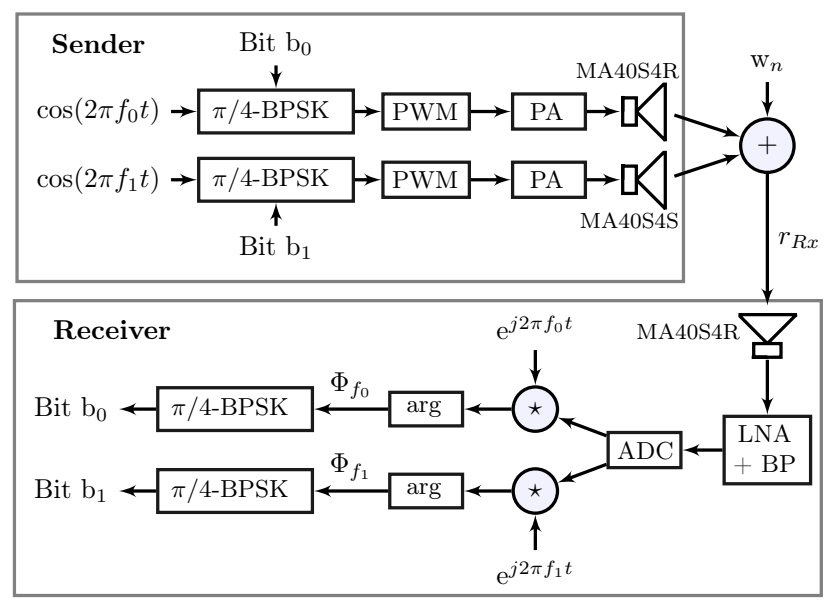

Figure 1. Schematic of the transmission system. The data bits $\left(b_{0}\right.$ and $b_{1}$ ) are mapped by $\pi / 4$-BSK, modulated by pulse width modulation (PWM) to the analog signal and gained by the power amplifier (PA). The receiver transforms the ultrasound signal into electric values and calculates after the analog-to-digital conversion (ADC) with the folding operation $*$ the phase angle of the signal and the demapper estimates the data bits.

tric transducer. In addition, the air is assumed to be linear and the attenuation difference between the neighboring frequencies is negligible due to the small frequency difference, the low amplitudes (below $120 \mathrm{~dB}$ SPL) and the interest in relative amplitudes. Moreover, the additive white Gaussian noise (AWGN) $w_{\mathrm{n}}$ adds the noise to the received signal. The low noise amplifier (LNA) with bandpass filter (BF) gains the received signal by a factor of 18 . The analog-to-digital converter (ADC) generates the digital data from the analog signal and the folding operation $*$ computes the phase angle $\Phi$ for both frequencies. At the end, the data are estimated from the demapped symbols.

The equivalent circuit for the piezoelectric transceiver is based on the Butterworth-van Dyke model (Van Dyke, 1928; Mason, 1935) see also the IRE standard (IRE, 1957). Figure 2 shows the equivalent circuit of the piezoelectric senders and the receiver. Thus, the piezoelectric transducer model includes a resistor, an inductance and two capacitances. In this work both transducers are used, the receiver (MA40S4R) and the sender (MA40S4S), for generating the ultrasound signal. However, only one transducer is used to receive the superposition of the signals from both devices.

\subsection{Sender}

The sender and receiver have the same equivalent circuit, for which each has different values. Therefore, the components are indexed by $k x$ with $k \in\{1,2\}$, whereby $k=1$ corresponds to the sender device and $k=2$ to the receiver device. The impedance of the sender is derived from the elements in series 


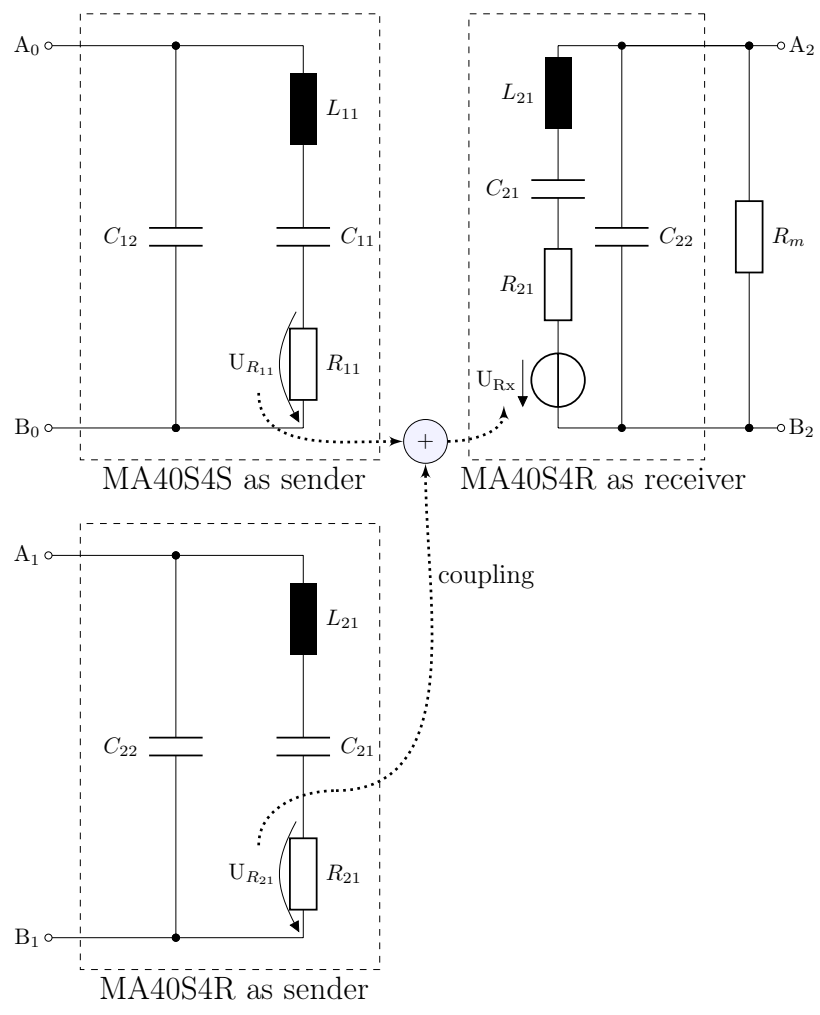

Figure 2. Equivalent circuit of the piezoelectric ultrasound senders (left) and receiver (right) with measurement resistor $R_{\mathrm{m}}$. The sender (MA40S4S) and receiver (MA40S4R) pair are both used as senders and only the receiver is used for the reception. The voltage at resistor $R_{11}$ and $R_{21}$ at the senders is directly coupled to the voltage source at the receiver as the received signal voltage $U_{R_{x}}$.

$Z_{T_{x}, \mathrm{~s}}(\omega, k)=R_{k 1}+\frac{1}{j \omega C_{k 1}}+j \omega L_{k 1}$

and the parallel capacitance $C_{k 2}$ to

$$
\begin{aligned}
Z_{T_{x}}(\omega, k) & =\frac{Z_{T_{x}, \mathrm{~s}} \cdot \frac{1}{j \omega C_{k 2}}}{Z_{T_{x}, \mathrm{~s}}+\frac{1}{j \omega C_{k 2}}} \\
& =\frac{R_{k 1}+\frac{1}{j \omega C_{k 1}}+j \omega L_{k 1}}{1+\frac{C_{k 2}}{C_{k 1}}+j \omega R_{k 1} C_{k 2}-\omega^{2} C_{k 2} L_{k 1}} .
\end{aligned}
$$

The transmitted acoustic power of the sender is proportional to the dissipated power of the resistor $R_{k 1}$. Moreover, the electrical impedance $Z_{T_{x}}$ is the analogy of the mechanical impedance $Z_{T_{x}, \mathrm{Me}}=|\boldsymbol{F}| /|\boldsymbol{v}|$ to model the mechanical behavior with the absolute value of the force $|\boldsymbol{F}|$ and the absolute value of the velocity $|\boldsymbol{v}|$ of the membrane surface (Lerch et al., 2008). Thus, the dissipated power $P_{\mathrm{El}}=U \cdot I$ in the resistor $R_{k 1}$ is equivalent to the mechanical power $P_{\mathrm{Me}}=\boldsymbol{F} \cdot \boldsymbol{v}$. Hence, the transfer function of the energy depends on the values of the capacitance $C_{k 1}$, the inductance $L_{k 1}$ and the resistor $R_{k 1}$ :

$$
\begin{aligned}
G_{T_{x}}(\omega, k) & =\frac{U_{T_{x}, R_{k 1}}}{U_{T_{x}} \text {,Input }}=\frac{R_{k 1}}{Z_{T_{x}}, \mathrm{~s}}, \\
& =\frac{j \omega C_{11} R_{k 1}}{1+j \omega C_{k 1} R_{k 1}-\omega^{2} C_{k 1} L_{k 1}} .
\end{aligned}
$$

\subsection{Receiver}

The measurement resistor $R_{\mathrm{m}}$ is connected in parallel to the piezoelectric transceiver (MA40S4R). The series and parallel resonance frequencies are independent of the measurement resistor. However, the measurement resistor influences the amplitude of the received signals. Hence, the usable carrier frequencies should be in the region of the resonance frequencies to receive signals with low loss and high amplitudes. The series impedance depends on the angular frequency $\omega=2 \pi f$ and includes the resistor $R_{21}$, series inductance $L_{21}$ and series capacitance $C_{21}$ :

$Z_{R_{x}, \mathrm{~s}}(\omega)=R_{21}+\frac{1}{j \omega C_{21}}+j \omega L_{21}$.

Furthermore, the voltage source in series with the resistor $R_{21}$, the capacitance $C_{21}$ and the inductance $L_{21}$ represents the transformation of the acoustic signal into an electric signal. The sink for the signal is the parallel impedance which includes the measurement resistor $R_{\mathrm{m}}$ and the parallel capacitance $C_{22}$ :

$Z_{R_{x}, \mathrm{p}}\left(R_{\mathrm{m}}, \omega\right)=\frac{1}{\frac{1}{R_{\mathrm{m}}}+j \omega C_{22}}=\frac{R_{\mathrm{m}}}{1+j \omega C_{22} R_{\mathrm{m}}}$.

Moreover, the transfer function from the source of the piezoelectric generator $U_{R_{x}}$ to the measurement resistor is

$G_{R_{x}}\left(R_{\mathrm{m}}, \omega\right)=\frac{Z_{R_{x}, \mathrm{p}}\left(R_{\mathrm{m}}, \omega\right)}{Z_{R_{x}, \mathrm{~s}}(\omega)+Z_{R_{x}, \mathrm{p}}\left(R_{\mathrm{m}}, \omega\right)}$.

\subsection{Parameter characterization}

For a numerical simulation of the presented model, the parameters of the piezoelectric transducers are calculated and characterized. Thus, the impedance of the receiver (Murata MA40S4R) and sender (Murata MA40S4S) devices is measured. Figure 3 shows the measured magnitudes of the impedances of both devices. The minimum of the magnitude corresponds to the series resonance and the maximum of the magnitude corresponds to the parallel resonance. Figure 4 shows the phase angle of the impedance of the piezoelectric sender and receiver. The phase crosses the abscissa at the resonance frequencies. Therefore, the imaginary part at the resonance frequencies is zero.

The values of $C_{21}$ and $L_{21}$ can be calculated approximately with the series resonance frequency $f_{\mathrm{s}}$ of the series resonance circuit (IRE, 1957)

$f_{\mathrm{s}}=\frac{1}{2 \pi \sqrt{L_{21} C_{21}}}$ 


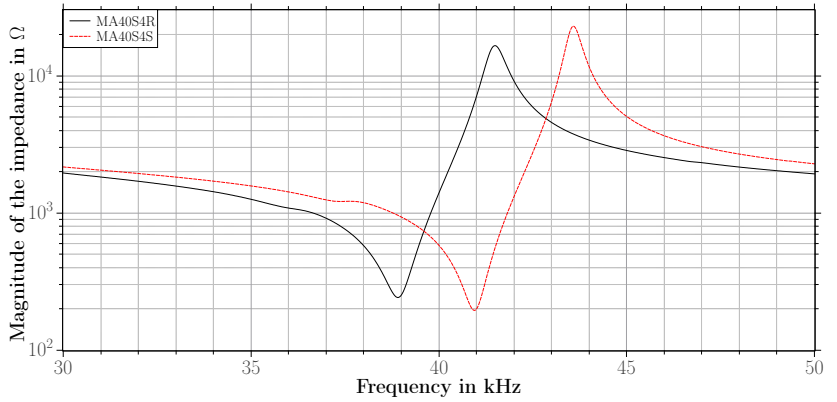

Figure 3. Impedance magnitude of the piezoelectric receiver and sender. The minimum magnitude corresponds to the series resonance and the maximum to the parallel resonance. The series resonance of the sender is near the parallel resonance of the receiver.

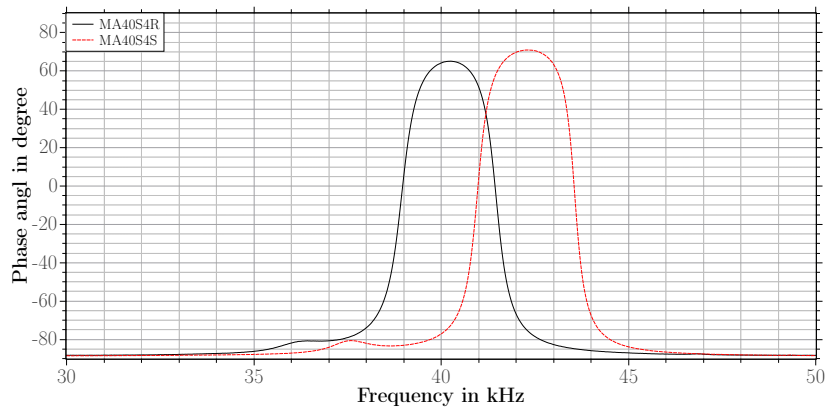

Figure 4. Impedance phase angle of the piezoelectric receiver and sender. The zero-crossings indicate the resonance frequencies.

and the resonance frequency of the parallel resonance circuit

$f_{\mathrm{p}}=\frac{1}{2 \pi} \sqrt{\frac{1}{L_{21} C_{21}}\left(1+\frac{C_{21}}{C_{22}}\right)}$.

Furthermore, the value of the parallel capacitance $C_{22}$ can be measured at low frequencies $\left(f \ll f_{\mathrm{s}}\right)$ or is given by the manufacturer (Mason, 1935). The capacitance $C_{22}$ is specified in the datasheet as $C_{22}=2.2 \mathrm{nF}$. Thus, the series capacitance can be computed with Eqs. (9) and (10) as

$C_{21}=C_{22}\left(\frac{f_{\mathrm{p}}^{2}}{f_{\mathrm{s}}^{2}}-1\right)$.

Furthermore, the value of the inductance with the series resonance frequency $f_{\mathrm{s}}$ and the capacitance $C_{21}$ is calculated with Eq. (11) as

$L_{21}=\frac{1}{\omega_{\mathrm{s}}^{2} C_{21}}$.

The quality factor $Q$ describes the damping of the resonance circuit depending on the capacitance and the resistor in the series resonance or on the resonance frequency and the $3 \mathrm{~dB}$ bandwidth (IRE, 1957):

$Q=\frac{1}{\omega_{\mathrm{s}} C_{21} R_{21}}=\frac{f_{\mathrm{s}}}{b}$.
Table 1. Measured and calculated values of the transceiver Murata MA40S4S and MA40S4R.

\begin{tabular}{lrr}
\hline & Sender MA40S4S, $k=1$ & Receiver MA40S4R, $k=2$ \\
\hline$f_{\mathrm{s}}$ & $40.8 \mathrm{kHz}$ & $38.8 \mathrm{kHz}$ \\
$f_{\mathrm{p}}$ & $43.5 \mathrm{kHz}$ & $41.5 \mathrm{kHz}$ \\
$R_{k 1}$ & $204 \Omega$ & $240 \Omega$ \\
$L_{k 1}$ & $43.6 \mathrm{mH}$ & $45.8 \mathrm{mH}$ \\
$C_{k 1}$ & $349 \mathrm{pF}$ & $367 \mathrm{pF}$ \\
$C_{k 2}$ & $2.55 \mathrm{nF}$ & $2.55 \mathrm{nF}$ \\
$Q$ & 54.8 & 46.6 \\
\hline
\end{tabular}

Table 1 shows the measured and calculated values for the receiver and sender transducers.

\section{Simulation}

The aim of the simulation is to find the optimal value of the measurement resistor $R_{\mathrm{m}}$ to receive both signals on two carrier frequencies $\left(f_{0}=38.8 \mathrm{kHz}\right.$ and $\left.f_{1}=40.8 \mathrm{kHz}\right)$ with the same signal strength. The received signal strength for every frequency depends on the value of the measurement resistor. In detail, a high value of the measurement resistor causes high amplitudes for signals received within the parallel resonance circuit. Indeed, a low value of the measurement resistor damps the parallel resonance circuit and the current in the series resonance circuit flows. Furthermore, the signal strength at the senders differs for the frequencies. Hence, the value has to be optimized regarding the ratio of the amplitudes $r=\frac{A_{\omega_{0}}}{A_{\omega_{1}}}$. $A_{\omega_{0}}$ and $A_{\omega_{1}}$ are the amplitudes of the signals at the implemented frequencies, which can be determined with a broad-band microphone. Moreover, the received signal $U_{R_{x}}$ consists of the transmitted voltage of both senders:

$U_{R_{x}}=U_{\mathrm{R}_{11}}+U_{\mathrm{R}_{21}}$.

Hence, the transfer function from the sender to the receiver is

$G_{\mathrm{m}}\left(R_{\mathrm{m}}, k\right)=G_{T_{x}}\left(R_{\mathrm{m}}\right) \cdot G_{R_{x}}(k)$

Therefore, the condition for optimal signal strength, with respect to the used frequencies $f_{0}$ and $f_{1}$, is

$\operatorname{Re}\left\{G_{\mathrm{m}}\left(R_{\mathrm{m}}, 1\right)\right\} \cdot r=\operatorname{Re}\left\{G_{\mathrm{m}}\left(R_{\mathrm{m}}, 2\right)\right\}$.

Thus, the optimal resistor follows through minimization:

$$
\begin{aligned}
& R_{\mathrm{m}, \text { opt }}= \\
& \quad \arg \min _{R_{\mathrm{m}}}\left|\operatorname{Re}\left\{G_{\mathrm{m}}\left(R_{\mathrm{m}}, 1\right)\right\} \cdot r-\operatorname{Re}\left\{G_{\mathrm{m}}\left(R_{\mathrm{m}}, 2\right)\right\}\right| .
\end{aligned}
$$

Figure 5 shows the numerical simulation results of the received voltage at the measurement resistor $R_{\mathrm{m}}$ for both frequencies $\left(f_{0}\right.$ and $\left.f_{1}\right)$ at different values of the measurement resistor. The curve of the voltage at the frequency $f_{1}$, which 


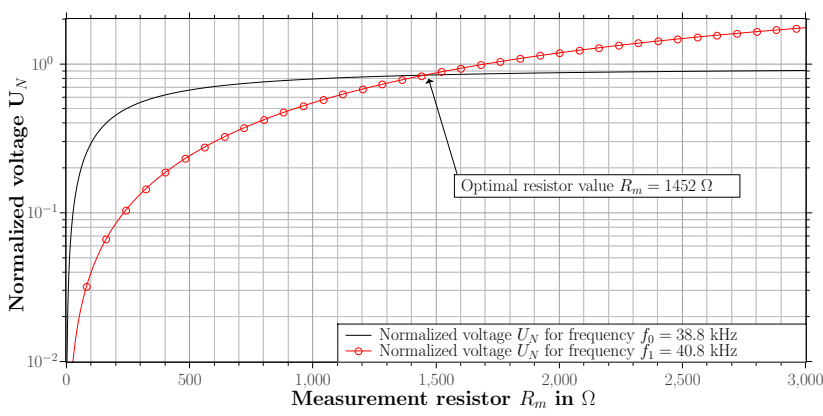

Figure 5. Graph of the voltages over different measurement resistor $R_{\mathrm{m}}$ values. The curves have different slopes and intersect for a measurement resistance of $R_{\mathrm{m}} \approx 1452 \Omega$.

is near the parallel resonance frequency, has a higher slope than the voltage of the frequency $f_{0}$. Furthermore, the voltage of the frequency $f_{0}$ (near to the series resonance) saturates to 0.9 . As a result, the optimal value of the measurement resistor is $R_{\mathrm{m}} \approx 1452 \Omega$, the intersection of both curves.

Moreover, a simulation of the transfer function for the optimal measurement resistor value was also performed. Figure 6 shows the simulation results for unmatched $\left(R_{\mathrm{m}}=1 \times\right.$ $\left.10^{6} \Omega\right)$ and matched $\left(R_{\mathrm{m}}=1452 \Omega\right)$ frequency response of the piezoelectric transmission system. An unoptimized system with $R_{\mathrm{m}}=1 \mathrm{M} \Omega$ requires a dynamic range $10 \mathrm{~dB}$ higher than an optimized system with $R_{\mathrm{m}}=1.452 \mathrm{k} \Omega$ to measure both signals with same resolution. Therefore, the ADC require $10 \mathrm{~dB}$ less dynamic range to measure both signals. Figure 7 shows the phase of the transfer function for the matched and unmatched receiver. The phase angle for $f_{0}=38.8 \mathrm{kHz}$ remains constant at about $0^{\circ}$ for both situations. However, the phase angle for $f_{1}=40.8 \mathrm{kHz}$ changes from about $-50^{\circ}$ for the unmatched case to $-80^{\circ}$ for the matched receiver. At the series resonance of $f_{0}=38.8 \mathrm{kHz}$ the imaginary part of the receiver device (MA40S4R) is about zero and the value of the resistor $R_{21}$ is dominant. Hence, the influence of the measurement resistor is marginal at the series resonance. Though, at the parallel resonance at $f_{1}=40.8 \mathrm{kHz}$ of the receiver device (MA40S4R), the imaginary part increases to the maximum value and the measurement resistor $R_{\mathrm{m}}$ in parallel becomes dominant. In addition, the measurement resistor $R_{\mathrm{m}}$ influences the phase and the amplitude of the signal at the frequency $f_{1}=40.8 \mathrm{kHz}$. As a result, the reduction of the measurement resistor $R_{\mathrm{m}}$ also reduces the real part and causes the phase shift of about $30^{\circ}$.

\section{Measurements}

The impedance and the transfer function of the system are measured to verify the simulation results and the capability of transmitting data. Therefore, the senders are placed together to act as a point source in line with the receiver within a distance of $30 \mathrm{~cm}$.

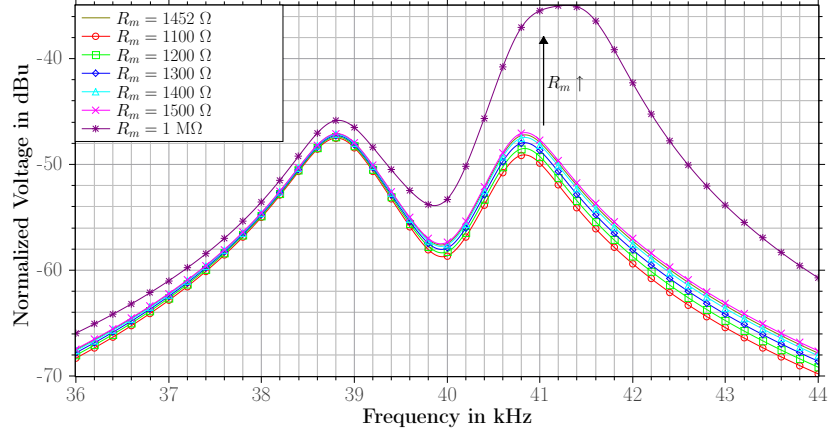

Figure 6. Graph of the simulated frequency response for matched $\left(R_{\mathrm{m}}=1452 \Omega\right)$ and unmatched $\left(R_{\mathrm{m}}=1 \times 10^{6} \Omega\right)$ piezoelectric receivers. The amplitude difference between the frequencies $f_{0}$ and $f_{1}$ for the unmatched receiver is about $10 \mathrm{~dB}$.

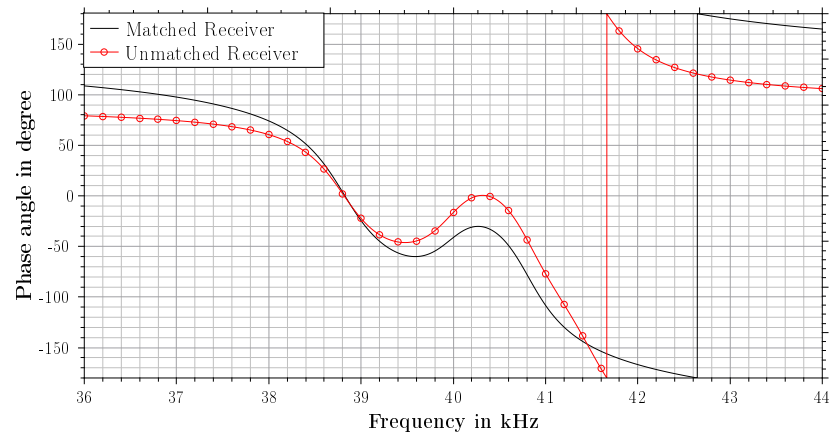

Figure 7. Graph of the simulated phase angle for matched $\left(R_{\mathrm{m}}=\right.$ $1452 \Omega)$ and unmatched $\left(R_{\mathrm{m}}=1 \times 10^{6} \Omega\right)$ piezoelectric receivers. The angle at the frequency $f_{0}=38.8 \mathrm{kHz}$ remains constant and the angle for the frequency $f_{1}=40.8 \mathrm{kHz}$ rotates by $30^{\circ}$.

Figure 8 shows the measured (colored lines with symbols) and simulated (black line) magnitude of the frequency response for different values of the measurement resistor. The simulation result, with $R_{\mathrm{m}}=1452 \Omega$, fits well with the measured result for $R_{\mathrm{m}}=1300 \Omega$. The magnitudes are similar for the frequencies $f_{0}=38.8 \mathrm{kHz}$ and $f_{1}=40.8 \mathrm{kHz}$. Thus, the model is a good approximation of the device. The measurement for $R_{\mathrm{m}}=1400 \Omega$ shows a difference of $0.5 \mathrm{~dB}$ of the amplitudes between the two frequencies.

The difference between the measured magnitudes in Fig. 8 for the measurement resistors $R_{\mathrm{m}}=1400 \Omega$ and $R_{\mathrm{m}}=$ $1300 \Omega$ is marginal. Hence the use of standard resistors with tolerances of about $5 \%$ is not critical for the matching.

Figure 9 shows the measured group delay of the signal from the senders to the receiver. The group delay is the same for the used frequencies $f_{0}=38.8 \mathrm{kHz}$ and $f_{1}=40.8 \mathrm{kHz}$ at about $1.36 \mathrm{~ms}$. Consequently, both frequencies can be used in OFDM to modulate separate carrier frequencies. 


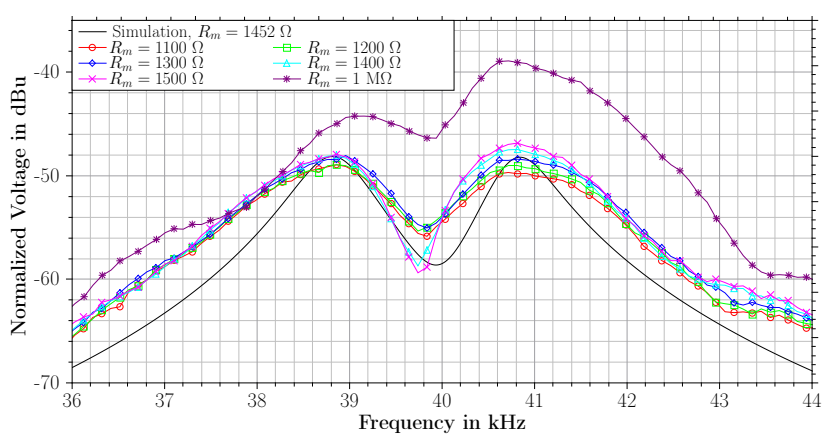

Figure 8. Measurement and simulation results of the voltage at the measurement resistor $R_{\mathrm{m}}$ for different values of the $R_{\mathrm{m}}$. The simulation (black line) with $R_{\mathrm{m}}=1454 \Omega$ coincides with the measured transfer function for the resistor value $R_{\mathrm{m}}=1300 \Omega$.

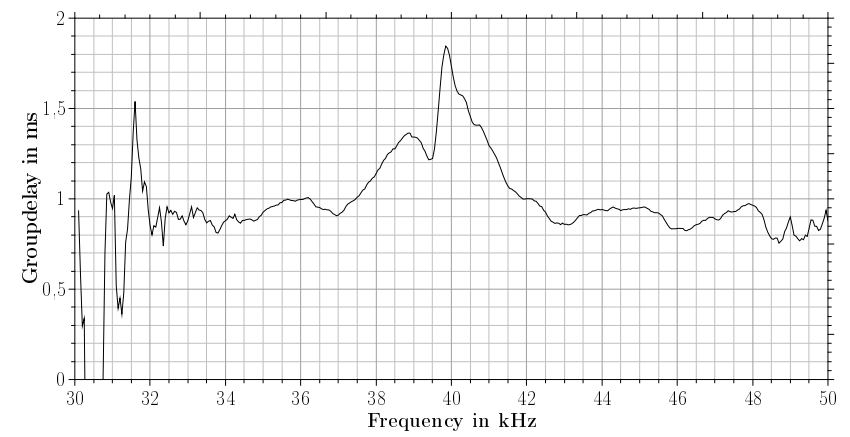

Figure 9. Measured group delay from the senders to the matched piezoelectric device. The delay is the same at the frequencies $f_{0}=$ $38.8 \mathrm{kHz}$ and $f_{1}=40.8 \mathrm{kHz}$ at $1.36 \mathrm{~ms}$.

The impedance of the receiver is measured to show the changes by the measurement resistor. Figure 10 shows the magnitude and Fig. 11 the phase angle of the impedance for the selected measurement resistor $R_{\mathrm{m}}=1 \mathrm{k} \Omega, R_{\mathrm{m}}=1.4 \mathrm{k} \Omega$ and $R_{\mathrm{m}}=1 \mathrm{M} \Omega$ (black curve). When increasing the measurement resistor, the phase angle is more flattened. Furthermore, the magnitude of the impedance of the parallel resonance circuit decreases to the value of the measurement resistor. The phase at the series resonance has a higher slope than at the parallel resonance. Therefore, the parallel resonance can be better adjusted to the used frequency. Hence, the low frequency signal $f_{0}$ has to be near the series resonance frequency, whereas the high frequency signal $f_{1}$ can be in the range of about $1 \mathrm{kHz}$ to the parallel resonance frequency of the piezoelectric transceiver.

The transmission system in Fig. 1 is matched by the optimal resistor with $1.4 \mathrm{k} \Omega$. The senders transmit two data bits ( $b_{0}=0$ and $b_{1}=1$ ) with the symbol duration of $1 \mathrm{~ms}$ and with the amplitude of $8 \mathrm{~V}_{\mathrm{pp}}$ over $1.3 \mathrm{~m}$. Hence, the phase shift is shifted for the carrier frequency $f_{0}$ by $\pi+\pi / 4 \approx$ -2.36 and for $f_{1}$ by $\pi / 4 \approx 0.79$. The first symbol is used as the reference symbol to calculate the phase angle difference to the second symbol. Figure 12 shows the received voltage

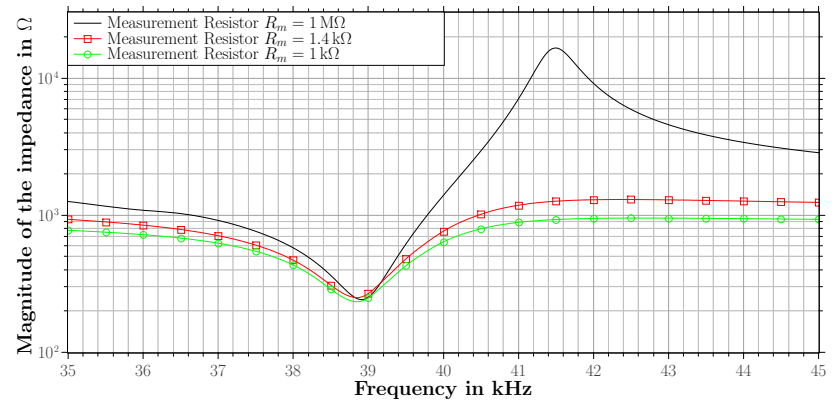

Figure 10. Measured magnitude for matched and unmatched piezoelectric devices (MA40S4R) for different measurement resistors. The impedance of the series resonance remains nearly constant for different resistor values. Indeed, the impedance of the parallel resonance decreases for lower resistor values.

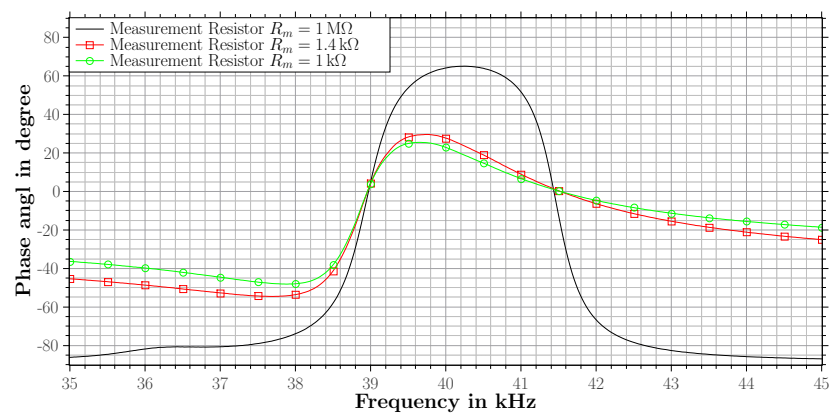

Figure 11. Measured phase angle for matched and unmatched piezoelectric devices (MA40S4R) for different measurement resistors. The phase angle for the series resonance remains constant for different resistor values at about $0^{\circ}$. However, the phase angle for the parallel resonance changes from $60^{\circ}$ for $R_{\mathrm{m}}=1 \mathrm{M} \Omega$ to $14^{\circ}$ for $R_{\mathrm{m}}=1 \mathrm{M} \Omega$.

at the receiver after the ADC. Before the first symbol there is the eigen oscillation of the receiver with the piezoelectric transducer and the bandpass filter. In the first symbol, the difference frequency of $2 \mathrm{kHz}$ can be recognized by the periodical change of the envelope with the period of $0.5 \mathrm{~ms}$. Moreover, the amplitude at $0.8 \mathrm{~ms}$ decreases nearly to zero, which indicates that the amplitudes of the carrier frequencies are similar. At the beginning of the second symbol, the phase angles change at both carrier frequencies and the amplitude decreases. After the first half of the symbol duration the phases angles are stabilized again and the envelope indicates the $2 \mathrm{kHz}$ difference frequency. As a result, the matching provides an optimal signal for data transmission with low complexity and effort. At the end of the transmission the senders and the receiver swing off, which is indicated as ringing.

Figure 13 shows the phase angle of the signal over time. The phase angle remains stable after the half of the first symbol. Thus, the phase is normalized to $0^{\circ}$ at the center of the first symbol. The phase angle varies at the begin- 


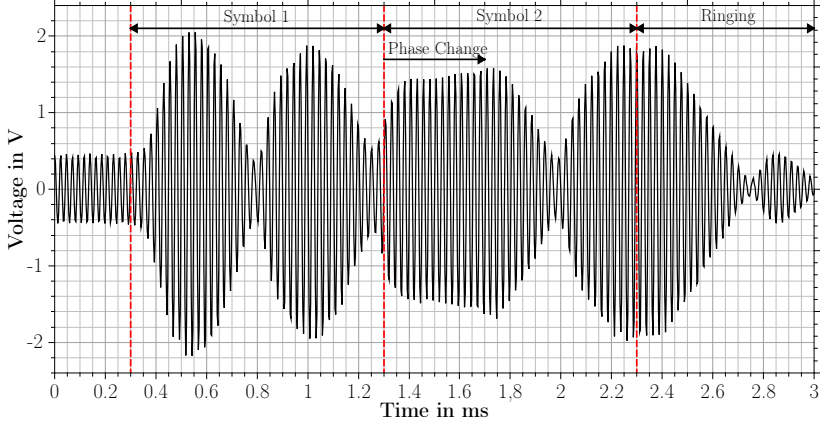

Figure 12. Measured voltage after the ADC of the receiver over time. The envelope changes with the difference frequency of $2 \mathrm{kHz}$ of the senders. Two symbols are transmitted with the phase change at the beginning of the second symbol.

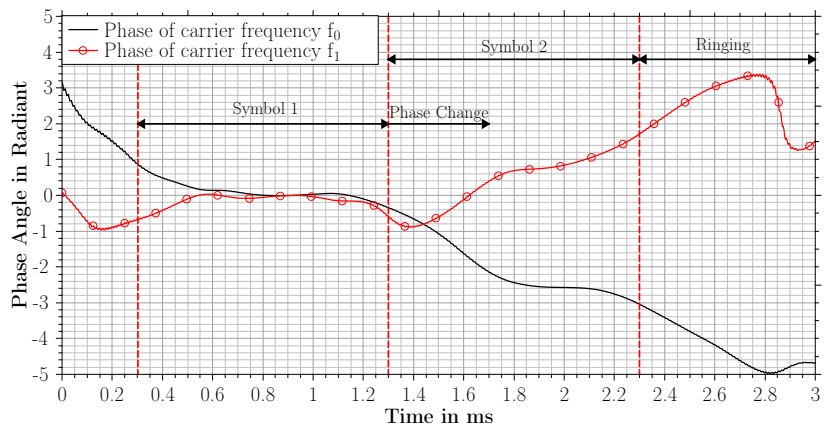

Figure 13. Measured phase angle of the received signal over time. The phase angles remain stable in the middle of the symbol. At the beginning of the second symbol the phases vary due to the phase shifts. The received phase angles $\Phi_{f_{0}} \approx-2.6$ and $\Phi_{f_{1}} \approx 0.8$ in the center of the second symbol coincide with the transmitted phase angles $\pi+\pi / 4 \approx-2.36$ for $f_{0}$ and $\pi / 4 \approx 0.79$ for $f_{1}$.

ning of the second symbol and settle down to $\Phi_{f_{0}} \approx-2.6$ and $\Phi_{f_{1}} \approx 0.8$. This coincides with the transmitted symbols $\pi+\pi / 4 \approx-2.36$ and $\pi / 4 \approx 0.79$. As a result, the phase error is $0.24 \mathrm{rad}(0.24 / \pi \approx 7.6 \%)$ for the carrier frequency $f_{0}$ and $0.01 \mathrm{rad}(0.01 / \pi \approx 0.3 \%)$ for the carrier frequency $f_{1}$. The phase error is due to noise and intersymbol interference (ISI) between the carrier frequencies. Consequently, the coded information in the phase shift can be decoded and mapped back to the data bits.

\section{Conclusion}

This work showed an optimization method for a piezoelectric receiver for multiple frequency applications based on an additional measurement resistor and multiple senders with different series resonances. This includes the determination of the model parameter and simulation of the model. Furthermore, a calculation method for the measurement resistor is introduced to use the series and parallel resonance of the transceivers. The measurement for the presented system with a measurement resistor of $R_{\mathrm{m}}=1300 \Omega$ for a signal with two different frequencies ( 38.8 and $40.8 \mathrm{kHz}$ ) shows the same amplitude. Moreover, the signals have the same amplitude and the same group delay $(1.36 \mathrm{~ms})$ for the matched transducer. As a result, the optimization reduces the required dynamic range of the ADC by $6 \mathrm{~dB}$. Hence, the ADC requires 1 bit fewer to ensure the same resolution for both carrier frequencies. Furthermore, the measurements validate our model and the optimization with numerical simulation. Data transmission works over two carrier frequencies with two senders and one receiver. Consequently, transmission systems based on multiple carrier frequencies may use piezoelectric transducers wherein the series resonance frequencies of the senders device lie in the range of the series and the parallel resonance frequency of the receiver device.

Acknowledgements. This work has been partly supported by the "Spitzencluster MicroTec Suedwest" and German Federal Ministry of Education and Research (BMBF) with the funding number 16SV5988.

Edited by: R. Lucklum

Reviewed by: three anonymous referees

\section{References}

Anderson, M., Cho, J. H., Richards, C. D., Bahr, D., and Richards, R.: A comparison of piezoelectric and electrostatic electromechanical coupling for ultrasonic transduction and power generation, in: 2005 IEEE Ultrasonics Symposium, Vol. 2, 950-955, doi:10.1109/ULTSYM.2005.1603008, 2005.

Bloomfield, P., Lo, W.-J., and Lewin, P.: Experimental study of the acoustical properties of polymers utilized to construct PVDF ultrasonic transducers and the acousto-electric properties of $\mathrm{PVDF}$ and $\mathrm{P}(\mathrm{VDF} / \mathrm{TrFE})$ films, IEEE Transactions on Ultrasonics, Ferroelectrics, and Frequency Control, 47, 1397-1405, doi:10.1109/58.883528, 2000.

Caliano, G., Savoia, A., Longo, C., Caronti, A., Pappalardo, M., Iula, A., and Rothmann, S.: cMUT sensor for applications as a wide-band acoustic receiver in the $\mathrm{MHz}$ range, in: 2010 IEEE Ultrasonics Symposium (IUS), 1869-1872, doi:10.1109/ULTSYM.2010.5935620, 2010.

Chang, R. W.: Synthesis of Band-Limited Orthogonal Signals for Multichannel Data Transmission, Bell Sys. Tech. J., 45, 17751796, doi:10.1002/j.1538-7305.1966.tb02435.x, 1966.

Ealo, J., Seco, F., and Jimenez, A.: Broadband EMFi-based transducers for ultrasonic air applications, IEEE T. Ultrason. Ferr., 55, 919-929, doi:10.1109/TUFFC.2008.727, 2008.

Ens, A., Höflinger, F., Wendeberg, J., Reindl, L. M., and Schindelhauer, C.: Indoor Positioning using Ultrasonic Waves with CSS and FSK Modulation for Narrow Band Channel, in: Int. Conf. on Indoor Positioning and Indoor Navigation, 2013.

Hosman, T., Yeary, M., and Antonio, J. K.: Design and Characterization of an MFSK-Based Transmitter/Receiver for Ultrasonic Communication Through Metallic Structures, IEEE T. Instrum. Meas., 60, 3767-3774, doi:10.1109/TIM.2011.2149270, 2011. 
Höher, P. A.: Grundlagen der digitalen Informationsübertragung Von der Theorie zu Mobilfunkanwendungen, Springer Vieweg, Wiesbaden, 2013.

IRE: IRE Standards on Piezoelectric Crystals-The Piezoelectric Vibrator: Definitions and Methods of Measurement, 1957, P. IRE, 45, 353-358, doi:10.1109/JRPROC.1957.278371, 1957.

ISO: ISO 9613-1:1993, Acoustics - Attenuation of sound during propagation outdoors - Part 1, 1993.

Jakes, W. C.: Microwave mobile communications, IEEE Press, Piscataway, NJ, 1993.

Lerch, R., Sessler, G., and Wolf, D.: Technische Akustik: Grundlagen und Anwendungen, Springer Bln, Berlin, 2008.

Mason, W.: An Electromechanical Representation of a Piezoelectric Crystal Used as a Transducer, P. IRE, 23, 1252-1263, doi:10.1109/JRPROC.1935.227273, 1935.
Oralkan, O., Ergun, A., Johnson, J., Karaman, M., Demirci, U., Kaviani, K., Lee, T., and Khuri-Yakub, B.: Capacitive micromachined ultrasonic transducers: next-generation arrays for acoustic imaging?, IEEE T. Ultrason. Ferr., 49, 1596-1610, doi:10.1109/TUFFC.2002.1049742, 2002.

Tränkler, H.-R. and Reindl, L.: Sensortechnik, Springer, New York, 2nd Edn., 2014.

Van Dyke, K.: The Piezo-Electric Resonator and Its Equivalent Network, P. IRE, 16, 742-764, doi:10.1109/JRPROC.1928.221466, 1928.

Weinstein, S. and Ebert, P.: Data Transmission by FrequencyDivision Multiplexing Using the Discrete Fourier Transform, IEEE T. Commun. Techn., 19, 628-634, doi:10.1109/TCOM.1971.1090705, 1971. 